\title{
DR Tauri: Temporal variability of the brightness distribution in the potential planet-forming region ${ }^{\star}$
}

\author{
R. Brunngräber ${ }^{1}$, S. Wolf ${ }^{1}$, Th. Ratzka ${ }^{2}$, and F. Ober ${ }^{1}$ \\ ${ }^{1}$ Institute of Theoretical Physics and Astrophysics, Christian-Albrechts-Universität zu Kiel, Leibnizstr. 15, 24118 Kiel, Germany \\ e-mail: rbrunngraeber@astrophysik. uni-kiel.de \\ ${ }^{2}$ Institute for Physics/IGAM, NAWI Graz, Karl-Franzens-Universität, Universitätsplatz 5/II, 8010 Graz, Austria
}

Received 8 June 2015 / Accepted 12 November 2015

\begin{abstract}
Aims. We investigate the variability of the brightness distribution and the changing density structure of the protoplanetary disk around DR Tau, a classical T Tauri star. DR Tau is known for its peculiar variations from the ultraviolet (UV) to the mid-infrared (MIR). Our goal is to constrain the temporal variation of the disk structure based on photometric and MIR interferometric data.

Methods. We observed DR Tau with the MID-infrared Interferometric instrument (MIDI) at the Very Large Telescope Interferometer (VLTI) at three epochs separated by about nine years, two months, respectively. We fit the spectral energy distribution and the MIR visibilities with radiative transfer simulations.

Results. We are able to reproduce the spectral energy distribution as well as the MIR visibility for one of the three epochs (third epoch) with a basic disk model. We were able to reproduce the very different visibility curve obtained nine years earlier with a very similar baseline (first epoch), using the same disk model with a smaller scale height. The same density distribution also reproduces the observation made with a higher spatial resolution in the second epoch, i.e. only two months before the third epoch.
\end{abstract}

Key words. stars: variables: T Tauri, Herbig Ae/Be - stars: individual: DR Tau - radiative transfer - techniques: interferometric protoplanetary disks

\section{Introduction}

Young stellar objects (YSOs) such as T Tauri stars gather material from their surrounding disk via accretion. It is commonly believed that the observed brightness variability of YSOs are due to a temporal increase of the stellar accretion rate that only lasts a limited amount of time (Hartmann \& Kenyon 1996; Mosoni et al. 2013). Various explanations for the temporal variations of accretion have been proposed: planet-disk interaction (Lodato \& Clarke 2004; Ruge et al. 2014), thermal or gravitational instability (Pringle 1981; Hartmann \& Kenyon 1996; Lodato \& Rice 2004, 2005; Zhu et al. 2009), interactions between the disk and a central binary (Pfalzner 2008), and the impact of the magnetic field (D'Angelo \& Spruit 2010). The flux variations can be traced from ultraviolet (UV) up to mid-infrared (MIR) wavelengths has been the subject of many photometric and spectroscopic studies in the past (Lorenzetti et al. 2009; Semkov et al. 2013; Antoniucci et al. 2014; Banzatti et al. 2014). With the rise of optical/infrared long baseline interferometry, one is potentially able to put stringent constraints on the region of the origin of the flux variations, even if not to spatially resolve it.

In this study, we investigate the variability of the protoplanetary disk of DR Tau, a classical T Tauri star. DR Tau is a member of the Taurus-Auriga molecular cloud at a distance of about 140 pc (Kenyon et al. 1994). It is known for its very strong, long- and short-term photometric and spectroscopic variability

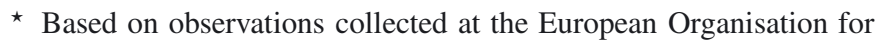
Astronomical Research in the Southern Hemisphere, Chile, under the programs 074.C-0342(A) and 092.C-0726(A,B). from the UV to the MIR (Kenyon \& Hartmann 1995; Alencar et al. 2001). Therefore the obtained parameters of the surrounding disk and the star itself vary with every new study. For instance, Banzatti et al. (2014) showed that the accretion luminosity can change by a factor of about two to three within less than two months by analysing the UV excess. Besides, age and spectral type of DR Tau could only be limited to be less than $4 \mathrm{Myr}$ and between K5 and K7, respectively (Muzerolle et al. 2003; Greaves 2004; Mohanty et al. 2005; Pontoppidan et al. 2011).

We present self-consistent disk models, simulated with the radiative transfer code MC3D (Wolf et al. 1999; Wolf 2003). These models reproduce highly spatially resolved interferometric data obtained with the MID-infrared Interferometric instrument (MIDI) at the VLTI at three different epochs. The measured visibilities show a clear change in the brightness distribution of the disk and, thus, we are able to determine the underlying variation of the dust density distribution. To reduce the degeneracies, a set of published photometric data is also used to model the spectral energy distribution (SED).

\section{Mid-infrared interferometric observations and data reduction}

The circumstellar disk of DR Tau was observed with MIDI (Leinert et al. 2003a,b; Morel et al. 2004) at the VLTI at three epochs. For all measurements the instrument was fed by two $8.2 \mathrm{~m}$ Unit Telescopes and the prism was used to disperse the light with a low resolution of about 30 . 
Table 1. Journal of our VLTI/MIDI observations.

\begin{tabular}{|c|c|c|c|c|c|c|c|}
\hline $\begin{array}{l}\text { Date of } \\
\text { observation }\end{array}$ & $\begin{array}{l}\text { Universal } \\
\text { time }\end{array}$ & Object & $\begin{array}{c}N \text {-band flux } \\
{[\mathrm{Jy}]}\end{array}$ & $\begin{array}{l}\text { Proj. I } \\
{[\mathrm{m}]}\end{array}$ & $\begin{array}{c}\text { Baseline } \\
\text { [deg] }\end{array}$ & $\begin{array}{l}\text { Interferometric } \\
\text { frames }\end{array}$ & $\begin{array}{l}\text { Photometric } \\
\text { frames }\end{array}$ \\
\hline \multicolumn{8}{|c|}{ U3-U4 (high sensitivity / prism) } \\
\hline \multicolumn{8}{|c|}{ E1 } \\
\hline 01-01-2005 & $01: 42-01: 54$ & HD $37160^{a, b}$ & 6.5 & 60.4 & 112.5 & $12000 \times 16 \mathrm{~ms}$ & $2 \times 4000 \times 16 \mathrm{~ms}$ \\
\hline 01-01-2005 & $02: 41-02: 48$ & DR Tau & - & 61.0 & 106.1 & $8000 \times 12 \mathrm{~ms}$ & $2 \times 1500 \times 12 \mathrm{~ms}$ \\
\hline 01-01-2005 & 02:51-03:00 & DR Tau & - & 60.4 & 105.6 & $8000 \times 12 \mathrm{~ms}$ & $2 \times 4000 \times 12 \mathrm{~ms}$ \\
\hline 01-01-2005 & $03: 19-03: 26$ & HD 31421 & 9.4 & 59.3 & 105.7 & $8000 \times 12 \mathrm{~ms}$ & $2 \times 1500 \times 12 \mathrm{~ms}$ \\
\hline 01-01-2005 & $03: 59-04: 07$ & HD 31421 & 9.4 & 55.2 & 104.4 & $8000 \times 12 \mathrm{~ms}$ & $2 \times 1500 \times 12 \mathrm{~ms}$ \\
\hline 01-01-2005 & $05: 24-05: 31$ & HD 49161 & 7.2 & 58.7 & 107.2 & $8000 \times 12 \mathrm{~ms}$ & $2 \times 1500 \times 12 \mathrm{~ms}$ \\
\hline
\end{tabular}

U2-U4 (high sensitivity / prism)

E2

\begin{tabular}{cccccccc}
$20-10-2013$ & $07: 10-07: 54$ & HD 25604 & 5.1 & 89.4 & 82.3 & $8000 \times 18 \mathrm{~ms}$ & $2 \times 4000 \times 18 \mathrm{~ms}$ \\
$20-10-2013$ & $07: 45-07: 52$ & HD 37160 & 6.5 & 86.7 & 84.2 & $8000 \times 18 \mathrm{~ms}$ & $2 \times 4000 \times 18 \mathrm{~ms}$ \\
$20-10-2013$ & $08: 01-08: 08$ & HD 31421 & 9.4 & 89.4 & 82.1 & $8000 \times 18 \mathrm{~ms}$ & $2 \times 2000 \times 18 \mathrm{~ms}$ \\
$20-10-2013$ & $08: 16-08: 24$ & DR Tau & - & 89.2 & 80.4 & $8000 \times 18 \mathrm{~ms}$ & $2 \times 4000 \times 18 \mathrm{~ms}$ \\
$20-10-2013$ & $08: 33-08: 41$ & HD 69142 & 5.7 & 82.0 & 55.5 & $8000 \times 18 \mathrm{~ms}$ & $2 \times 4000 \times 18 \mathrm{~ms}$ \\
\hline
\end{tabular}

U3-U4 (high sensitivity / prism)

E3

\begin{tabular}{cccccccc}
\hline $20-12-2013$ & $01: 02-01: 08$ & HD 31421 & 9.4 & 57.2 & 117.9 & $8000 \times 18 \mathrm{~ms}$ & $2 \times 2000 \times 18 \mathrm{~ms}$ \\
$20-12-2013$ & $01: 30-01: 37$ & DR Tau & - & 60.6 & 115.2 & $8000 \times 18 \mathrm{~ms}$ & $2 \times 4000 \times 18 \mathrm{~ms}$ \\
$20-12-2013$ & $01: 54-02: 03$ & HD 33554 & $7.7^{c}$ & 60.4 & 115.0 & $8000 \times 18 \mathrm{~ms}$ & $2 \times 6000 \times 18 \mathrm{~ms}$ \\
$20-12-2013$ & $02: 55-03: 03$ & HD 25604 $^{b}$ & 5.1 & 60.1 & 104.3 & $8000 \times 18 \mathrm{~ms}$ & $2 \times 4000 \times 18 \mathrm{~ms}$ \\
$20-12-2013$ & $03: 41-03: 48$ & HD 37160 $^{a}$ & 6.5 & 62.4 & 108.9 & $8000 \times 18 \mathrm{~ms}$ & $2 \times 4000 \times 18 \mathrm{~ms}$ \\
\hline
\end{tabular}

Notes. The length and position angle of the projected baselines have been determined from the fringe tracking sequence. ${ }^{(a)}$ Spectrophotometric calibrator; ${ }^{(b)}$ peculiar instrumental visibility; ${ }^{(c)} \mathrm{N}$-band flux taken from CalVin.

The data were reduced with the MIA+EWS package ${ }^{1}$. The visibilities shown were computed with MIA that is based on the analysis of the power spectrum. The visibility shown for the first night is the mean of the two calibrated measurements. The standard error is 0.07 on average. All visibilities have been calibrated by all the calibrators taken in the same night and with the same mode as the science target. The stability of the transfer function is reflected by the errors shown together with the visibilities. Calibrators with peculiar visibilities have been ignored and are indicated in the journal of observations; see Table 1. A comparison of the MIA results with the results obtained with EWS confirm the visibilities. For the last night the EWS visibility even tends to be lower up to about 0.1 at short $N$-band wavelengths. The EWS is based on a coherent analysis of the interferometric fringe signal (Jaffe 2004). The differential phases of our observations do not significantly differ from $0^{\circ}$. The maximum differential phase is at about $10^{\circ}$ for E2 but strongly depends on the used calibrator and thus the uncertainties are very high. Besides, differential phases equal to zero do not necessarily mean that there are no asymmetries in the target because linear phase terms cannot be probed (Ratzka et al. 2009).

The data used for our analysis were taken in January 2005, October 2013, and December 2013. Additional measurements were made in October and November 2004 and in December 2013, but were excluded due to their low quality. The data set presented and modelled by Schegerer et al. (2009) has been re-reduced. The calibrated visibilities for the three epochs are shown in Fig. 1.

\footnotetext{
1 http://wWw.strw.leidenuniv.nl/ nevec/MIDI/
}

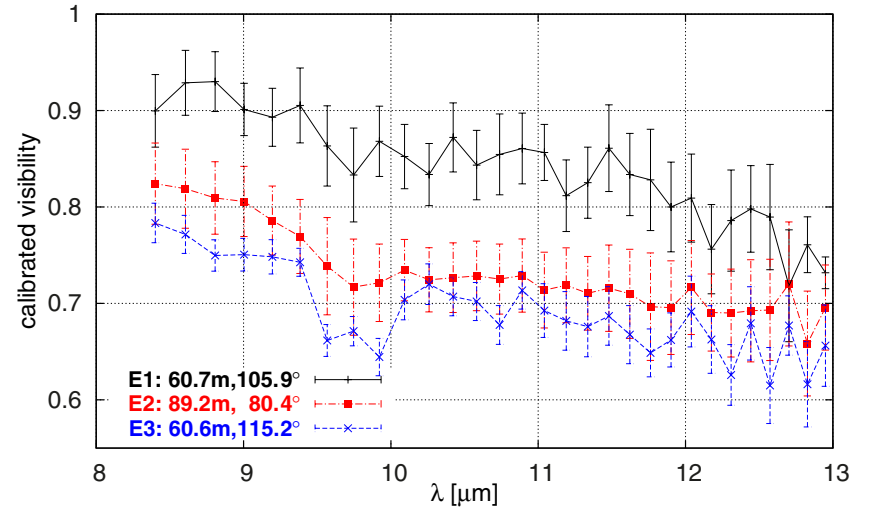

Fig. 1. Calibrated MIR visibility of DR Tau for the three different epochs January 2005 (E1), October 2013 (E2), and December 2013 (E3).

\section{Data analysis and disk modelling}

The properties of our target DR Tau are listed in Table 2. Previous photometric measurements are given in Table 3.

\subsection{Temporal variability}

Interferometry: the visibilities obtained at the first epoch in January $2005\left(E 1^{2}\right)$ differ significantly from those made at E3

\footnotetext{
2 In the following, we denote the epochs 1, 2, and 3 as E1, E2, and E3, respectively.
} 
Table 2. Stellar properties of DR Tau.

\begin{tabular}{llr}
\hline \hline Parameter & Value & Ref. \\
\hline RA (J2000) & $04 \mathrm{~h} 47 \mathrm{~m} 06^{\mathrm{s}} .2$ & 1 \\
Dec (J2000) & $+16^{\circ} 58^{\prime} 42^{\prime \prime} 8$ & 1 \\
Distance & $140 \mathrm{pc}$ & 2 \\
Visual extinction & $1.6 \mathrm{mag}$ & 3 \\
Spectral type & K5-7 & 3,4 \\
Stellar mass & $0.4-1 M_{\odot}$ & 4,5 \\
Total luminosity & $1-5.5 L_{\odot}$ & $3,5,6,7$ \\
Age & $\lesssim 4 \mathrm{Myr}$ & 8,9 \\
\hline
\end{tabular}

References. (1) 2MASS All Sky Catalog of point sources (Cutri et al. 2003); (2) Kenyon et al. (1994); (3) Muzerolle et al. (2003); (4) Pontoppidan et al. (2011); (5) Isella et al. (2009); (6) Banzatti et al. (2014); (7) Eisner et al. (2014); (8) Mohanty et al. (2005); (9) Greaves (2004).

Table 3. Photometric data of DR Tau.

\begin{tabular}{lcr}
\hline \hline$\lambda[\mu \mathrm{m}]$ & Flux $[\mathrm{Jy}]$ & Ref. \\
\hline 0.36 & $0.050 \pm 0.033$ & 1 \\
0.44 & $0.061 \pm 0.035$ & 1 \\
0.55 & $0.095 \pm 0.047$ & 1 \\
0.64 & $0.149 \pm 0.076$ & 1 \\
0.78 & $0.26 \pm 0.15$ & 1 \\
1.25 & $0.46 \pm 0.01$ & 2 \\
1.65 & $0.78 \pm 0.04$ & 2 \\
2.17 & $1.22 \pm 0.02$ & 2 \\
3.6 & $1.86 \pm 0.20$ & 3 \\
4.5 & $1.89 \pm 0.15$ & 3 \\
5.8 & $2.34 \pm 0.02$ & 4 \\
8 & $1.92 \pm 0.01$ & 4 \\
12 & $3.16 \pm 0.03$ & 5 \\
25 & $4.30 \pm 0.05$ & 5 \\
60 & $5.51 \pm 0.04$ & 5 \\
100 & $5.73 \pm 0.63$ & 6 \\
200 & $4.10 \pm 0.85$ & 7 \\
450 & $2.38 \pm 0.17$ & 6 \\
600 & $0.61 \pm 0.05$ & 8 \\
729 & $0.40 \pm 0.08$ & 9 \\
850 & $0.533 \pm 0.007$ & 6 \\
1056 & $0.23 \pm 0.02$ & 9 \\
1300 & $0.109 \pm 0.011$ & 10 \\
\hline
\end{tabular}

References. (1) Kenyon \& Hartmann (1995); (2) 2MASS All Sky Catalog of point sources (Cutri et al. 2003); (3) Robitaille et al. (2007); (4) Hartmann et al. (2005); (5) Weaver \& Jones (1992); (6) Andrews \& Williams (2005); (7) ISO Data Archive; (8) Mannings \& Emerson (1994); (9) Beckwith \& Sargent (1991); (10) Isella et al. (2009).

in December 2013, although the projected baseline lengths (BL) and orientations are nearly the same. These observations indicate changes of the brightness distribution and, hence, the underlying density distribution in the innermost regions of the disk ( $\lesssim 4 \mathrm{AU})$. Thus, these two observations cannot be reproduced with a static disk model. Possible reasons could be a general brightness increase of the disk due to a variable accretion rate onto the central star or azimuthal brightness asymmetries that have significantly changed their position between both epochs of observation. Local brightness asymmetries on the scale of several dozens to hundreds of AU have already been observed in the case of various protoplanetary disks, e.g. GM Aur (Schneider et al. 2003), FS Tau (Hioki et al. 2011), IM Lupi (Pinte et al. 2008). However, in contrast to these observations only long-baseline interferometry in the optical/infrared provides the angular resolution so far to trace regions, so close to the central star that the orbital motion on the scale of months to years has to be taken into account in the data analysis. In the very near future, ALMA will provide a similar angular resolution in the submm/mm wavelength range.

On the other hand, the visibility obtained at E2 (October 2013) with the much longer BL of about $90 \mathrm{~m}$ is higher than the measurement with $\mathrm{BL} \approx 60 \mathrm{~m}$ two months later (E3). A higher visibility means more compact structure in the case of simple structures. Thus, an observation with a longer BL, i.e. tracing higher spatial frequencies, result in a lower visibility, which is not the case here. Of course, we observed at two different baseline orientations, which at first glance might explain this counterintuitive scenario. A static, rotationally symmetric, inclined disk, observed at slightly different orientations can produce different visibilities if one observation is almost aligned with the minor or major axis of the disk. This difference becomes larger with higher orientation difference and higher disk inclination, however, our observations were made at an orientation difference of only $35^{\circ}$ and there is no evidence of a disk inclination larger than $60^{\circ}$ (Kenyon et al. 1994; Alencar et al. 2001; Brown et al. 2013; Eisner et al. 2014); see also Sect. 4. Thus, it could either be a hint of short-term variations at the same timescale as the UV variations observed by Banzatti et al. (2014) or for a non-rotationally symmetric disk, i.e. disk structures seen at different phases of their orbit. In summary, our interferometric observations indicate the presence of a non-axisymmetric structure of the disk and/or temporal variations of the disk structure in mid-infrared bright regions of the disk.

Photometry: for a moderately inclined disk, we expect to observe a silicate emission feature at $\approx 10 \mu \mathrm{m}$ and a smooth SED without depressions or peaks. In contrast to the adjacent photometric data points, the Spitzer/IRAC data suggest a lack of flux at $8 \mu \mathrm{m}$; see Table 3. Moreover, this is the same wavelength region MIDI is sensitive to, and the non-stable visibility suggests a non-stable flux. Thus, it is possible that this photometric Spitzer observation was made during a more quiet phase of DR Tau. Because of this, we exclude the IRAC data point from our fitting procedure.

\subsection{Model set-up}

Earlier studies: many different values have been published for the orientation of the disk, described by the disk inclination $i$ and the disk position angle PA. The inclination ranges from $9^{\circ}$ (Pontoppidan et al. 2011) to $60^{\circ}$ (Akeson et al. 2005) and the position angle is presumed to be between $75^{\circ}$ (Eisner et al. 2014) and $180^{\circ}$ (Andrews \& Williams 2005). The disk is rather massive, $M_{\text {disk }} \approx 0.01-0.1 M_{\odot}$ (Akeson et al. 2005; Robitaille et al. 2007; Schegerer et al. 2009; Ricci et al. 2010), and the inner radius has been determined to be less than $0.1 \mathrm{AU}$ through analysing the emission of $\mathrm{CO}$ and $\mathrm{H}_{2} \mathrm{O}$ (Brown et al. 2013; Banzatti et al. 2014; Eisner et al. 2014).

Disk structure: Schegerer et al. (2009) also fit photometric and interferometric data for DR Tau but only for one epoch (E1). Their results are used as a starting point for our simulation, which also applies to the Ansatz of Shakura \& Sunyaev (1973) for the density distribution. It is given by

$\varrho(r, z)=\varrho_{0}\left(\frac{R_{\star}}{r}\right)^{\alpha} \exp \left[-\frac{1}{2}\left(\frac{z}{h(r)}\right)^{2}\right]$, 
where $r$ and $z$ are the usual cylindrical coordinates, $R_{\star}$ the stellar radius, and $h(r)$ the scale height:

$h(r)=h_{100}\left(\frac{r}{100 \mathrm{AU}}\right)^{\beta}$

The two exponents $\alpha$ and $\beta$ describe the radial density profile and disk flaring, respectively. Assuming a coupling of temperature and surface density, the number of free parameters decreases by one (Shakura \& Sunyaev 1973):

$\alpha=3\left(\beta-\frac{1}{2}\right)$.

This simple disk model has already been used successfully for fitting spatially resolved and unresolved multi-wavelength observations of several protoplanetary disks (Wolf et al. 2003; Ratzka et al. 2009; Sauter \& Wolf 2011; Madlener et al. 2012; Gräfe et al. 2013; Liu et al. 2013).

Dust properties: the dust in our set-up is a mixture of $62.5 \%$ astronomical silicate and $37.5 \%$ graphite (12.5\% parallel and $25 \%$ perpendicular) and has particle sizes between $5 \mathrm{~nm}$ and $250 \mathrm{~nm}$. The grain size distribution is given by the MRN distribution (Mathis et al. 1977), i.e. follows a power law, $n(s) \propto s^{-3.5}$, where $n \cdot \mathrm{d} s$ are the number of particles in the radius interval $[s, s+\mathrm{d} s]$.

Heating sources: the central star is treated as a black body with a fixed value for the total luminosity of $L_{\mathrm{tot}}=L_{\star}+L_{\mathrm{acc}}=1.9 L_{\odot}$ (Muzerolle et al. 2003; Schegerer et al. 2009; Eisner et al. 2014). Further, we set the stellar effective temperature to $T_{\star}=4050 \mathrm{~K}$ (Mohanty et al. 2005). The disk around DR Tau is actively accreting (Robitaille et al. 2007; Edwards et al. 2013; Eisner et al. 2014). Therefore, we also assume an additional accretion heating of the disk. To implement this, we use a very simple, static accretion model, by adding a second point-like, black-body source in the centre of our disk. The properties of this accretion source, i.e. temperature and truncation radius, are fixed in this study to $T_{\text {accr }}=8000 \mathrm{~K}$ and $R_{\text {trunc }}=5 R_{\star}$, respectively. These values were already successfully used to model other TTSs (Akeson et al. 2005; Schegerer et al. 2008, 2009).

To calculate the dust temperature distribution and the corresponding SED and mid-infrared visibilities, we use the radiativetransfer code MC3D (Wolf et al. 1999; Wolf 2003).

\subsection{Modelling}

Fitting procedure: in the standard fitting approach, the photometric and interferometric data were used simultaneously. The SED is a degenerate quantity and more than just one adequate density distribution could reproduce the observations. The visibility curves are then used to reduce these degeneracies, since they are only sensitive to small-scale structures in the interior of the disk. According to Sect. 3.1, this approach is not suitable for our observations. The visibilities obtained at the three epochs cannot be reproduced by a disk with only one parameter set. Thus, we independently fit all four sets, SED + three visibilities. Due to the set-up of our global model, a resulting parameter set based on a visibility is only valid for the innermost brightness distribution and does not represent the entire disk.

Parameter space and fitness quality: to explore the parameter space, we use an iteratively refined grid search. The boundaries of the parameter space and the overall number of used values for
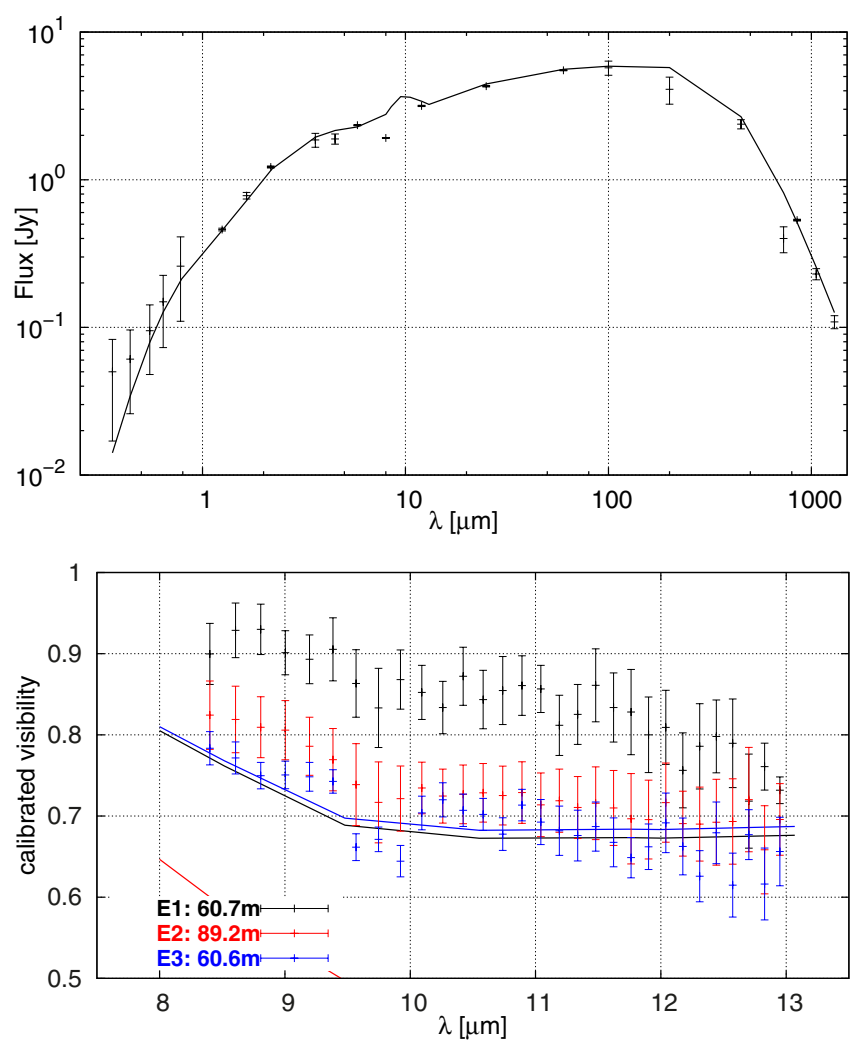

Fig. 2. Best SED fit with corresponding MIR visibilities. The three visibility epochs are shown in different colours (black: first, red: second, blue: third). Observations: with uncertainties; model: solid lines.

Table 4. Boundaries of parameter space.

\begin{tabular}{lllr}
\hline \hline Disk parameter & Min. value & Max. value & $n$ \\
\hline$L_{\star}\left[L_{\odot}\right]$ & 0.25 & 1.9 & 9 \\
$L_{\text {accr }}\left[L_{\odot}\right]$ & 0 & 1.65 & 9 \\
$M_{\text {dust }}\left[M_{\odot}\right]$ & $8 \times 10^{-4}$ & $3 \times 10^{-3}$ & 2 \\
$R_{\text {in }}[\mathrm{AU}]$ & 0.065 & 2 & 23 \\
$R_{\text {out }}[\mathrm{AU}]$ & 70 & 350 & 5 \\
$\beta$ & 0.65 & 1.3 & 14 \\
$h_{100}[\mathrm{AU}]$ & 10 & 20 & 13 \\
Inclination $i\left[^{\circ}\right]$ & 8 & 62 & 14 \\
Position Angle $\mathrm{PA}\left[{ }^{\circ}\right]$ & 0 & 175 & 36 \\
\hline
\end{tabular}

each parameter are shown in Table 4. To compare all individual models, we calculate $\chi^{2}$ for each resulting SED

$\chi^{2}=\sum_{i=1}^{m}\left(\frac{F_{\mathrm{SED}}\left(\lambda_{i}\right)-F_{\text {model }}\left(\lambda_{i}\right)}{\sigma_{\mathrm{SED}}\left(\lambda_{i}\right)}\right)^{2}$,

where $m$ is the number of wavelengths in Table 3 and $\sigma_{\text {SED }}$ the photometric uncertainties. To evaluate the visibilities, we apply a similar approach. Because of the huge amount of time needed to calculate a radiation map with sufficient resolution, we do not calculate the visibilities and thus the $\chi^{2}$ value for every measured wavelength bin given by the spectral resolution of MIDI, but at seven wavelengths between 8 and $13 \mu \mathrm{m}$.

\section{Results}

In the upper part of Fig. 2, our best SED fit is shown. We excluded the Spitzer/IRAC data point at $8 \mu \mathrm{m}$ from the fitting process (see Sect. 3.1). The properties of this disk are listed in the 
Table 5. Model parameters for our best fits.

\begin{tabular}{lcccc}
\hline \hline \multirow{2}{*}{ Disk parameter } & \multicolumn{3}{c}{ Best fit value } & Schegerer et al. (2009) \\
\hline & SED & E3 & E1 + E2 & Model III \\
\hline$L_{\star}\left[L_{\odot}\right]$ & 0.9 & 0.9 & 0.9 & 0.9 \\
$L_{\text {accr }}\left[L_{\odot}\right]$ & 1.0 & 1.0 & 1.0 & 1.0 \\
$T_{\star}[\mathrm{K}]$ & $4050^{a}$ & $4050^{a}$ & $4050^{a}$ & 4050 \\
$T_{\text {accr }}[\mathrm{K}]$ & $8000^{a}$ & $8000^{a}$ & $8000^{a}$ & 8000 \\
$M_{\text {dust }}\left[M_{\odot}\right]$ & $3 \times 10^{-3 b}$ & $3 \times 10^{-3 b}$ & $3 \times 10^{-3 b}$ & $1 \times 10^{-3}$ \\
$R_{\text {in }}[\mathrm{AU}]$ & 0.111 & $0.065^{c}$ & $0.065^{c}$ & 0.05 \\
$R_{\text {out }}[\mathrm{AU}]$ & 200 & 90 & $350^{d}$ & 90 \\
$\beta$ & 1.025 & 1.025 & 1.025 & 0.75 \\
$h_{100}[\mathrm{AU}]$ & 18 & 17.5 & $10^{c}$ & 15 \\
Inclination $\left.i{ }^{\circ}\right]$ & 44 & 44 & 44 & 20 \\
\hline
\end{tabular}

Notes. In the last column, the results of Schegerer et al. (2009) are also printed for comparison. ${ }^{(a)}$ Fixed values; ${ }^{(b)}$ only two values included; ${ }^{(c)}$ lower boundary; ${ }^{(d)}$ upper boundary.

second column of Table 5. These values are in agreement with other DR Tau studies; cf. Banzatti et al. (2014), Eisner et al. (2014), Isella et al. (2009), Schegerer et al. (2009), Muzerolle et al. (2003). These parameters, however, cover a rather wide range, which could be directly related to the short- and longterm variations of the disk structure. Interestingly, the best-fit parameters result in mid-infrared visibilities, which are in agreement with those obtained in E3, although the visibilities were not considered during the SED fitting process (see lower part of Fig. 2).

The corresponding visibilities in Fig. 2 are plotted for a $\mathrm{PA} \approx 50^{\circ}$ from north to east for the major axis of the disk. As predicted in Sect. 3.1, the two visibilities with nearly the same set-up from January 2005 (E1) and December 2013 (E3) cannot be reproduced with the same disk parameter values. Although the PA is a free parameter here, the baseline orientation difference of just $10^{\circ}$ is too small to produce this difference in the visibility. Because of the small inclination of the disk, the visibility is not very sensitive to changes in the PA. The calculated visibilities with $\mathrm{BL}=60.6 \mathrm{~m}$ (blue solid line) vary only between $5 \%$ and $12 \%$ due to changes in the PA. This uncertainty is also confirmed by previous DR Tau studies: Pontoppidan et al. (2011) found a PA of about $0^{\circ}$, Isella et al. (2009) found $100^{\circ}$, Brown et al. (2013) published $140^{\circ}$ and Akeson et al. (2005) derived $\mathrm{PA}=160^{\circ} \pm 55^{\circ}$.

In Fig. 3 our best result for the visibility obtained at epoch E3 is shown. As you can see, both the visibility curve for E3 and the SED are very similar to those in Fig. 2. The major differences in both set-ups are only in the inner radius, which changed from $0.111 \mathrm{AU}$ to $0.065 \mathrm{AU}$, and the outer radius, which changed from $200 \mathrm{AU}$ to $90 \mathrm{AU}$. The larger outer radius of the first model results in more cold dust, which increases the flux in the far-IR and (sub-)mm regime and matches the photometry better at these wavelengths. Furthermore, MIDI measurements are not sensitive to the outer disk because of the limited FoV of the interferometer. The other parameters are the same or very similar for both models, especially inclination and flaring, and thus the radial density profile is almost unchanged (see Eq. (3)).

A reasonable scenario for the found differences between the visibilities of E1 and E3 could be fast changes in the accretion luminosity, as mentioned in Sect. 1. To investigate this, we used the disk set-up that reproduces the SED best, and set $L_{\text {accr }}=1.9 L_{\odot}$, resulting in a total luminosity of $L_{\text {tot }}=2.8 L_{\odot}$. As a consequence, the dust heating is stronger at all distances and disk layers. This leads to a SED that is higher at all wavelengths
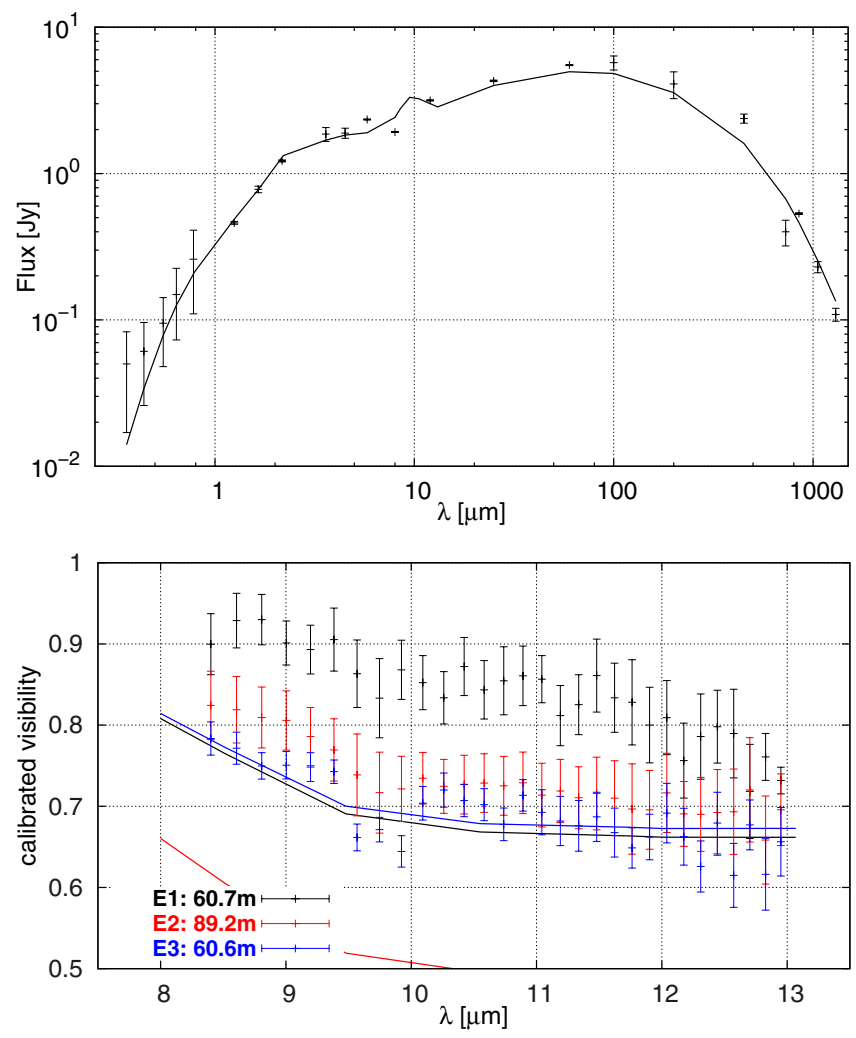

Fig. 3. Best fit for third epoch visibility with corresponding SED. The three visibility epochs are shown in different colours (black: first, red: second, blue: third). Observations: with uncertainties; model: solid lines.

and to visibilities that drop by about $10 \%$ for all three baselines. If the accretion luminosity is decreased or even completely eliminated $\left(L_{\text {tot }}=L_{\star}=0.9 L_{\odot}\right)$, the visibilities increase up to $20 \%$ for the longest wavelengths. Therefore, the lack of accretion cannot reproduce our observations as the visibilities are still too low for $\mathrm{E} 1$ and $\mathrm{E} 2$, and hence a different disk set-up is needed.

Our third parameter set reproduces the observations of E1 and E2 simultaneously, although they are almost nine years apart (see Fig. 4). If we compare the parameters of this best-fit model with those of the two previous models, we find that only the scale height and outer radius have changed and that flaring and inclination are again the same. This disk set-up is only valid for 

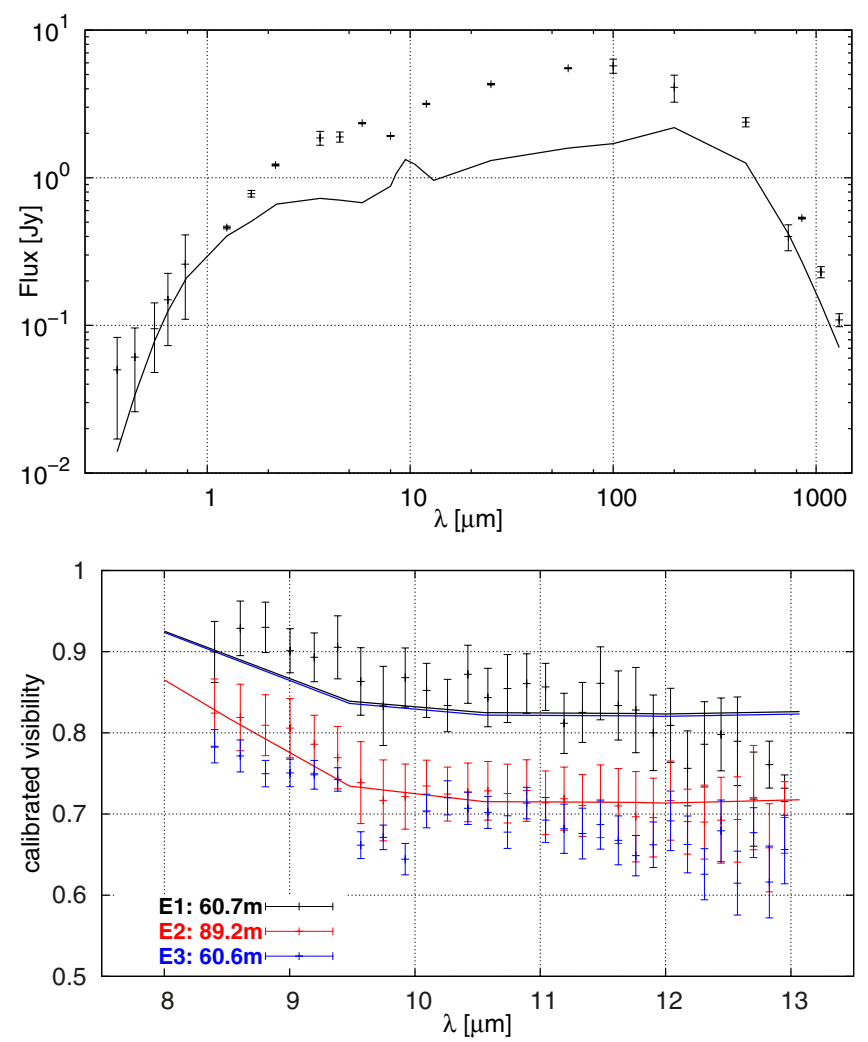

Fig. 4. Best fit for first and second epoch visibility with corresponding SED. The three visibility epochs are shown in different colours (black: first, red: second, blue: third). Observations: with uncertainties; model: solid lines.

small stellar separations of $r \lesssim 10$ AU. More distant regions are not covered by the MIR visibilities and hence not considered by the fitting process. Because of the huge outer radius of model 3, the density in the hot, inner region is lower. In contrast, the scale height is decreased by nearly $50 \%$, which significantly increases the density in the midplane of the disk, resulting in a higher optical depth and a more compact emission. Hence, the visibility in the MIR is higher compared to the other two models and fits the observations. Owing to our purely global disk model, the SED in Fig. 4 does not reproduce the observations and is too low for nearly all wavelengths. With the much smaller scale height, the disk of model 3 is very compact, resulting in a higher optical depth. Thus, the dust heating is less efficient, resulting in a reduced flux.

\section{Conclusion}

We presented MIR visibilities of the T Tauri star DR Tau obtained with MIDI/VLTI. We modelled the SED and the individual mid-infrared visibility curves at three epochs to constrain the underlying variation in the disk density structure. Our simulations suggest that the hot, inner regions of the disk were more compact in January 2005 (E1) than they were in December 2013 (E3), especially in the layers close to the midplane. Since these two observations were made with nearly the same baseline length and position angle, it is possible that the structural difference is due to a local density variation, such as a density clump observed at different stages of its orbit. If this is true, the relevant region can be approximated by assuming a Keplerian orbit. If this hypothetical clump revolved once or twice within these nine years, its orbit must be about 2.5 or $4 \mathrm{AU}$, respectively.
For a stellar distance of $140 \mathrm{pc}$, this is exactly the resolution of MIDI with a BL $\approx 60 \mathrm{~m}$. Since we cannot distinguish between $\mathrm{PA}=0^{\circ}$ and $\mathrm{PA}=180^{\circ}$, we detect the same fringe pattern twice per orbit. Although it is very unlikely, it cannot be completely ruled out that we observed the same density structure at first and second epoch. Considering a circular orbit of $2.5 \mathrm{AU}$ with a period of $\approx 4.5$ years, in two months the clump would have moved $\approx 13^{\circ}-14^{\circ}$ on its orbit. Together with the PA difference of $35^{\circ}$ between $\mathrm{E} 2$ and $\mathrm{E} 3$, it is also possible to explain the non-detection at $\mathrm{E} 3$.

However, we are aware of the fact that the photometric data set (see Table 3 ) is based on observations made at different epochs, i.e. at different stages of temporal evolution of the disk. Detailed studies over different timescales are necessary to achieve a better understanding of the temporal variations of protoplanetary disks and thus the underlying disk physics. With this pioneering study, we want to stress this problem, which has been commonly ignored in most disk modelling studies so far.

With the second generation instrument MATISSE on the VLTI, a much better uv-coverage will be possible than with MIDI (Lopez et al. 2014). The MATISSE instrument will measure up to six visibilities at once with four telescopes, which is crucial for a consistent view on the disk at one certain point in time. Moreover, studying the temporal evolution of substructures could constrain physical processes in the potential planetforming region (Kley et al. 2009; Flock et al. 2015; Bitsch et al. 2015). With existing MIDI data, new observations will enable us to trace disk evolution on timescales of more than 15 years. Even though MATISSE will cover a much wider wavelength range than MIDI ( $L, M$, and $N$ band), it is mandatory to observe protoplanetary disks with other interferometric instruments in different wavelength regimes also. High-spatial-resolution observations in the NIR and (sub-)mm, such as obtained with PIONIER/VLTI, AMBER/VLTI, and ALMA, will allow us to constrain the density distribution for dust at different temperatures, i.e. different disk regions.

Acknowledgements. This research was funded through the DFG grant: WO 857/13-1. This publication makes use of data products from the Two Micron All Sky Survey, which is a joint project of the University of Massachusetts and the Infrared Processing and Analysis Center/California Institute of Technology, funded by the National Aeronautics and Space Administration and the National Science Foundation.

\section{References}

Akeson, R. L., Walker, C. H., Wood, K., et al. 2005, ApJ, 622, 440

Alencar, S. H. P., Johns-Krull, C. M., \& Basri, G. 2001, AJ, 122, 3335

Andrews, S. M., \& Williams, J. P. 2005, ApJ, 631, 1134

Antoniucci, S., Giannini, T., Li Causi, G., \& Lorenzetti, D. 2014, ApJ, 782, 51

Banzatti, A., Meyer, M. R., Manara, C. F., Pontoppidan, K. M., \& Testi, L. 2014, ApJ, 780, 26

Beckwith, S. V. W., \& Sargent, A. I. 1991, ApJ, 381, 250

Bitsch, B., Johansen, A., Lambrechts, M., \& Morbidelli, A. 2015, A\&A, 575, A28

Brown, L. R., Troutman, M. R., \& Gibb, E. L. 2013, ApJ, 770, L14

Cutri, R. M., Skrutskie, M. F., van Dyk, S., et al. 2003, 2MASS All Sky Catalog of point sources

D’Angelo, C. R., \& Spruit, H. C. 2010, MNRAS, 406, 1208

Edwards, S., Kwan, J., Fischer, W., et al. 2013, ApJ, 778, 148

Eisner, J. A., Hillenbrand, L. A., \& Stone, J. M. 2014, MNRAS, 443, 1916

Flock, M., Ruge, J. P., Dzyurkevich, N., et al. 2015, A\&A, 574, A68

Gräfe, C., Wolf, S., Guilloteau, S., et al. 2013, A\&A, 553, A69

Greaves, J. S. 2004, MNRAS, 351, L99

Hartmann, L., \& Kenyon, S. J. 1996, ARA\&A, 34, 207

Hartmann, L., Megeath, S. T., Allen, L., et al. 2005, ApJ, 629, 881

Hioki, T., Itoh, Y., Oasa, Y., Fukagawa, M., \& Hayashi, M. 2011, PASJ, 63, 543

Isella, A., Carpenter, J. M., \& Sargent, A. I. 2009, ApJ, 701, 260 
R. Brunngräber et al.: DR Tau: Temporal variability

Jaffe, W. J. 2004, in New Frontiers in Stellar Interferometry, ed. W. A. Traub, SPIE Conf. Ser., 5491, 715

Kenyon, S. J., \& Hartmann, L. 1995, ApJS, 101, 117

Kenyon, S. J., Dobrzycka, D., \& Hartmann, L. 1994, AJ, 108, 1872

Kley, W., Bitsch, B., \& Klahr, H. 2009, A\&A, 506, 971

Leinert, C., Graser, U., Przygodda, F., et al. 2003a, Ap\&SS, 286, 73

Leinert, C., Graser, U., Waters, L. B. F. M., et al. 2003b, in Interferometry for Optical Astronomy II, ed. W. A. Traub, SPIE Conf. Ser., 4838, 893

Liu, Y., Wang, H.-C., Wolf, S., \& Madlener, D. 2013, RA\&A, 13, 841

Lodato, G., \& Clarke, C. J. 2004, MNRAS, 353, 841

Lodato, G., \& Rice, W. K. M. 2004, MNRAS, 351, 630

Lodato, G., \& Rice, W. K. M. 2005, MNRAS, 358, 1489

Lopez, B., Lagarde, S., Jaffe, W., et al. 2014, The Messenger, 157, 5

Lorenzetti, D., Larionov, V. M., Giannini, T., et al. 2009, ApJ, 693, 1056

Madlener, D., Wolf, S., Dutrey, A., \& Guilloteau, S. 2012, A\&A, 543, A81

Mannings, V., \& Emerson, J. P. 1994, MNRAS, 267, 361

Mathis, J. S., Rumpl, W., \& Nordsieck, K. H. 1977, ApJ, 217, 425

Mohanty, S., Jayawardhana, R., \& Basri, G. 2005, ApJ, 626, 498

Morel, S., Ballester, P., Bauvir, B., et al. 2004, in New Frontiers in Stellar Interferometry, ed. W. A. Traub, SPIE Conf. Ser., 5491, 1666

Mosoni, L., Sipos, N., Ábrahám, P., et al. 2013, A\&A, 552, A62

Muzerolle, J., Calvet, N., Hartmann, L., \& D’Alessio, P. 2003, ApJ, 597, L149
Pfalzner, S. 2008, A\&A, 492, 735

Pinte, C., Padgett, D. L., Ménard, F., et al. 2008, A\&A, 489, 633

Pontoppidan, K. M., van Dishoeck, E., Blake, G. A., et al. 2011, The Messenger, 143,32

Pringle, J. E. 1981, ARA\&A, 19, 137

Ratzka, T., Schegerer, A. A., Leinert, C., et al. 2009, A\&A, 502, 623

Ricci, L., Testi, L., Natta, A., et al. 2010, A\&A, 512, A15

Robitaille, T. P., Whitney, B. A., Indebetouw, R., \& Wood, K. 2007, ApJS, 169, 328

Ruge, J. P., Wolf, S., Uribe, A. L., \& Klahr, H. H. 2014, A\&A, 572, L2

Sauter, J., \& Wolf, S. 2011, A\&A, 527, A27

Schegerer, A. A., Wolf, S., Ratzka, T., \& Leinert, C. 2008, A\&A, 478, 779

Schegerer, A. A., Wolf, S., Hummel, C. A., Quanz, S. P., \& Richichi, A. 2009, A\&A, 502, 367

Schneider, G., Wood, K., Silverstone, M. D., et al. 2003, AJ, 125, 1467

Semkov, E. H., Peneva, S. P., Munari, U., et al. 2013, A\&A, 556, A60

Shakura, N. I., \& Sunyaev, R. A. 1973, A\&A, 24, 337

Weaver, W. B., \& Jones, G. 1992, ApJS, 78, 239

Wolf, S. 2003, Comput. Phys. Comm., 150, 99

Wolf, S., Henning, T., \& Stecklum, B. 1999, A\&A, 349, 839

Wolf, S., Padgett, D. L., \& Stapelfeldt, K. R. 2003, ApJ, 588, 373

Zhu, Z., Hartmann, L., Gammie, C., \& McKinney, J. C. 2009, ApJ, 701, 620 